\title{
Li isotopes in metal-poor halo dwarfs: a more and more complicated story
}

\author{
Monique Spite and François Spite \\ GEPI, Observatoire de Paris, 92195 Meudon Cedex, CNRS UMR 8111 \\ email: monique.spite@obspm.fr, francois.spite@obspm.fr
}

\begin{abstract}
The nuclei of the lithium isotopes are fragile, easily destroyed, so that, at variance with most of the other elements, they cannot be formed in stars through steady hydrostatic nucleosynthesis.

The ${ }^{7} \mathrm{Li}$ isotope is synthesized during primordial nucleosynthesis in the first minutes after the Big Bang and later by cosmic rays, by novae and in pulsations of AGB stars (possibly also by the $\nu$ process). ${ }^{6} \mathrm{Li}$ is mainly formed by cosmic rays. The oldest (most metal-deficient) warm galactic stars should retain the signature of these processes if, (as it had been often expected) lithium is not depleted in these stars. The existence of a "plateau" of the abundance of ${ }^{7} \mathrm{Li}$ (and of its slope) in the warm metal-poor stars is discussed. At very low metallicity $([\mathrm{Fe} / \mathrm{H}]<-2.7 \mathrm{dex})$ the star to star scatter increases significantly towards low $\mathrm{Li}$ abundances. The highest value of the lithium abundance in the early stellar matter of the Galaxy $\left(\log \epsilon(\mathrm{Li})=\mathrm{A}\left({ }^{7} \mathrm{Li}\right)=2.2 \operatorname{dex} \dagger\right)$ is much lower than the the value $(\log \epsilon(\mathrm{Li})=2.72)$ predicted by the standard Big Bang nucleosynthesis, according to the specifications found by the satellite WMAP. After gathering a homogeneous stellar sample, and analysing its behaviour, possible explanations of the disagreement between Big Bang and stellar abundances are discussed (including early astration and diffusion). On the other hand, possibilities of lower productions of ${ }^{7} \mathrm{Li}$ in the standard and/or non-standard Big Bang nucleosyntheses are briefly evoked.

A surprisingly high value $\left(\mathrm{A}\left({ }^{6} \mathrm{Li}\right)=0.8 \mathrm{dex}\right)$ of the abundance of the ${ }^{6} \mathrm{Li}$ isotope has been found in a few warm metal-poor stars. Such a high abundance of ${ }^{6} \mathrm{Li}$ independent of the mean metallicity in the early Galaxy cannot be easily explained. But are we really observing ${ }^{6} \mathrm{Li}$ ?
\end{abstract}

Keywords. Stars: abundances, Population II - nucleosynthesis - early universe

\section{Introduction}

\subsection{A first view}

In the eighties and the nineties, the abundance of ${ }^{7} \mathrm{Li}$ in the warm $(5700<$ Teff $<$ $6500 \mathrm{~K})$ metal-poor dwarfs was found constant, independent of the temperature and of the metallicity, at least in the interval $-2.8<[\mathrm{Fe} / \mathrm{H}]<-1.8$ : Spite \& Spite $(1982 \mathrm{a}$, and 1982b) and subsequent papers (e.g. Spite, Spite and Maillard 1984, Hobbs \& Thorburn 1991, Molaro, Primas and Bonifacio 1995, Spite et al. 1996, Bonifacio and Molaro 1997, Smith et al. 1998). Later works, published up to 2005, are vividly reviewed in the section 2 of Charbonnel and Primas (2005) : the small differences between authors are discussed.

This "universal" behaviour of lithium suggested that ${ }^{7} \mathrm{Li}$ observed in the old metal poor dwarfs was synthesized during primordial nucleosynthesis (when the Universe was only a few minutes old) and that lithium, although a very fragile element, had survived unaltered in the atmosphere of warm metal-poor dwarfs. In the so called "standard" stellar models (i. e. without diffusion and mixing), the Li depletion is negligible (Deliyannis et al. 1990, Pinsonneault et al. 1992). However, already at that time, several theoreticians of stellar

$\dagger$ In the literature the lithium abundance, is noted indifferently by $\log \epsilon(\mathrm{Li})$ or by $\mathrm{A}(\mathrm{Li})$; both notations are in logarithmic scale of number of atoms where $\log \epsilon(\mathrm{H})=\mathrm{A}(\mathrm{H})=12$ 
atmospheres thought that it was difficult to admit that the abundance of lithium in the atmosphere of the old very metal-poor dwarfs had remained unchanged during 13 billions years. Reciprocally, it was also difficult to explain a strictly uniform lithium depletion in the warm metal-poor dwarfs, whatever their temperature, mass and metallicity.

The primordial abundance of ${ }^{7} \mathrm{Li}$ had been then largely identified with the stellar abundance found on the plateau, the value being between $\log \epsilon(\mathrm{Li})=2.0$ and $\log \epsilon(\mathrm{Li})=2.3$ depending on the temperature scale adopted by the authors. Moreover, taking into account the errors of measurement and the uncertainties of the observed abundances, the simultaneous production of all the light elements $\left({ }^{4} \mathrm{He},{ }^{3} \mathrm{He}, \mathrm{D}\right.$ and $\left.\mathrm{Li}\right)$ by the Big Bang, could be explained.

In spite of the obvious difficulty of disentangling the ${ }^{6} \mathrm{Li}$ and ${ }^{7} \mathrm{Li}$ profiles in stellar spectra, attempts were made at finding the abundance of ${ }^{6} \mathrm{Li}$ : it was possible only in a handful of metal-poor dwarfs. For the first time Smith, Lambert, \& Nissen (1993), claimed a positive detection of ${ }^{6} \mathrm{Li}$ in a metal-poor star: $\mathrm{HD} 84937([\mathrm{Fe} / \mathrm{H}] \approx-2.1)$. Later Nissen et al. (1999) observed ${ }^{6} \mathrm{Li}$ in 2 metal-poor stars out of five; see also Cayrel et al. (1999). The existence of ${ }^{6} \mathrm{Li}$ (more fragile that ${ }^{7} \mathrm{Li}$ ) in some warm metal-poor dwarfs reinforced the idea that ${ }^{7} \mathrm{Li}$ is not depleted in such stars.

\subsection{A second step}

Two astronomical satellites brought new points of view :

- in 1997, Hipparcos has provided accurate parallaxes for numerous stars, improving the determination of the parameters of the stellar atmospheres (and enabling to discriminate dwarfs and subgiants).

- in 2003, WMAP has determined (Bennett et al. 2003) the physical conditions of the Big Bang especially a precise value for the baryons to photons ratio : $\eta$, leading to precise values of the abundances of the light elements produced in the standard Big Bang, including lithium. This Li abundance was found much higher than the abundance measured in the warm metal-poor dwarfs and thus the interest for a precise re-determination of the lithium abundance was reactivated.

\section{The lithium isotopes in the matter of the early Galaxy}

\subsection{Formation of Lithium}

A peculiarity of lithium is that it is very fragile and destroyed at "low" temperatures. Unlike most of the other elements, when it is synthesized inside the stars by hydrostatic nucleosynthesis, it is immediately destroyed. Lithium is formed :

- during the Big Bang (primordial nucleosynthesis) $\left({ }^{7} \mathrm{Li}\right.$ and negligible amount of ${ }^{6} \mathrm{Li}$ ).

- by spallation (cosmic rays or in superbubbles: mainly ${ }^{6} \mathrm{Li}$ )

- possibly by the " $\nu$ " process in type II supernovae $\left({ }^{7} \mathrm{Li}\right)$

Pulsations of AGB stars and novae explosions can also enrich the interstellar medium in ${ }^{7} \mathrm{Li}$, but not in the early times of the Galaxy.

\subsection{Primordial abundance of ${ }^{7} \mathrm{Li}$}

The ${ }^{7} \mathrm{Li}$ observed in the atmospheres of the very old galactic stars has been mainly formed by the primordial nucleosynthesis. Cosmic rays and $\nu$ process in massive supernovae could produce a small increase of $\epsilon\left({ }^{7} \mathrm{Li}\right)$ with metallicity (a slope).

In the standard Big-Bang, the production of lithium depends only on the baryons to photons ratio $\eta$. This ratio has been deduced, with precision, from the measurements 
of the cosmic microwave background radiation by the satellite WMAP : Bennett et al. (2003), Spergel et al. (2007), Cyburt et al. (2008) : $\eta=6.23 \pm 0.1710^{-10}$.

Then, the primordial lithium abundance is $\log \epsilon\left({ }^{7} \mathrm{Li}\right)=2.72 \pm 0.06$ for $\log \epsilon(\mathrm{H})=12$ (see also Komatsu et al. 2009, and Iocco et al. 2009, for the discussion of the uncertainties).

\subsection{Depletion of lithium in the atmosphere of the stars}

It is generally admitted that the abundances measured in the atmospheres of unevolved stars are representative of the abundances in the material from which the star has been originally formed.

However different phenomena are known to affect the superficial abundance of lithium during the life of the star.

Lithium is a very fragile element destroyed as soon as the temperature is higher than $2.510^{6} \mathrm{~K}$ for ${ }^{7} \mathrm{Li}$ and even $2.010^{6} \mathrm{~K}$ for ${ }^{6} \mathrm{Li}$.

If there is some mixing between the surface of the star and the hot deep layers, little by little lithium is destroyed and disappears from the atmosphere of the star. In giants, Li is strongly diluted after the first dredge-up. Even in dwarfs lithium is often depleted: in the atmosphere of the Sun after $4.5 \mathrm{Gyr}$, the lithium abundance is only $\log \epsilon(\mathrm{Li})=1.03$ (Caffau et al., this volume) although in meteorites $\log \epsilon(\mathrm{Li})$ reaches $\approx 3.25$ (a value representative of the abundance of lithium in the material that formed the Sun). However in the warm metal-poor dwarfs/turnoff stars, mixing is not as deep as in solar metallicity stars, and lithium could have been preserved.

Lithium is also supposed to slowly settle down into the stars by diffusion (gravitational settling), but the diffusion can be easily thwarted by turbulent mixing.

\section{The behaviour of ${ }^{7} \mathrm{Li}$ in the most metal poor stars in the Milky Way}

The difference between the lithium abundance predicted by BBN + WMAP and the mean value observed in the atmosphere of the old galactic dwarfs, leads to a redetermination of the lithium abundance, with an effort towards better temperature determinations: the determination of lithium abundance is very sensitive to the choice of the temperature of the stellar atmosphere. Moreover, the precise behaviour of $\log \epsilon(\mathrm{Li})$ vs. temperature and metallicity and also the scatter around the mean relations may give a clue about the exact mechanism of lithium depletion.

Since 2005 several papers about the lithium abundance in very metal-poor stars were published. They all try to determine very carefully the temperatures of the stellar atmospheres, using high quality data: it is essential to discriminate between intrinsic scatter and determinations errors.

-Charbonnel \& Primas (2005) have determined the temperatures from a very precise photometry (uvby) associated with the Alonso et al. (1996) calibration and taking into account very carefully the reddening of the stars. Meléndez et al. (2010 and this symposium) had a similar approach but they used a new implementation of the infrared flux method to determine the temperatures from multiband IR photometry.

-Boesgaard et al. (2005) and Hosford et al. (2009) based their analysis on excitation temperature (the abundances from the Fe I lines must be independent of the excitation potential of the line).

-All the other authors used the wings of the hydrogen lines (mainly H $\alpha$ ) to determine the temperatures: Asplund et al. (2006), Bonifacio et al. (2007), González Hernández et al. (2008), García Pérez et al. (2008, 2009), Aoki et al.( 2009). 

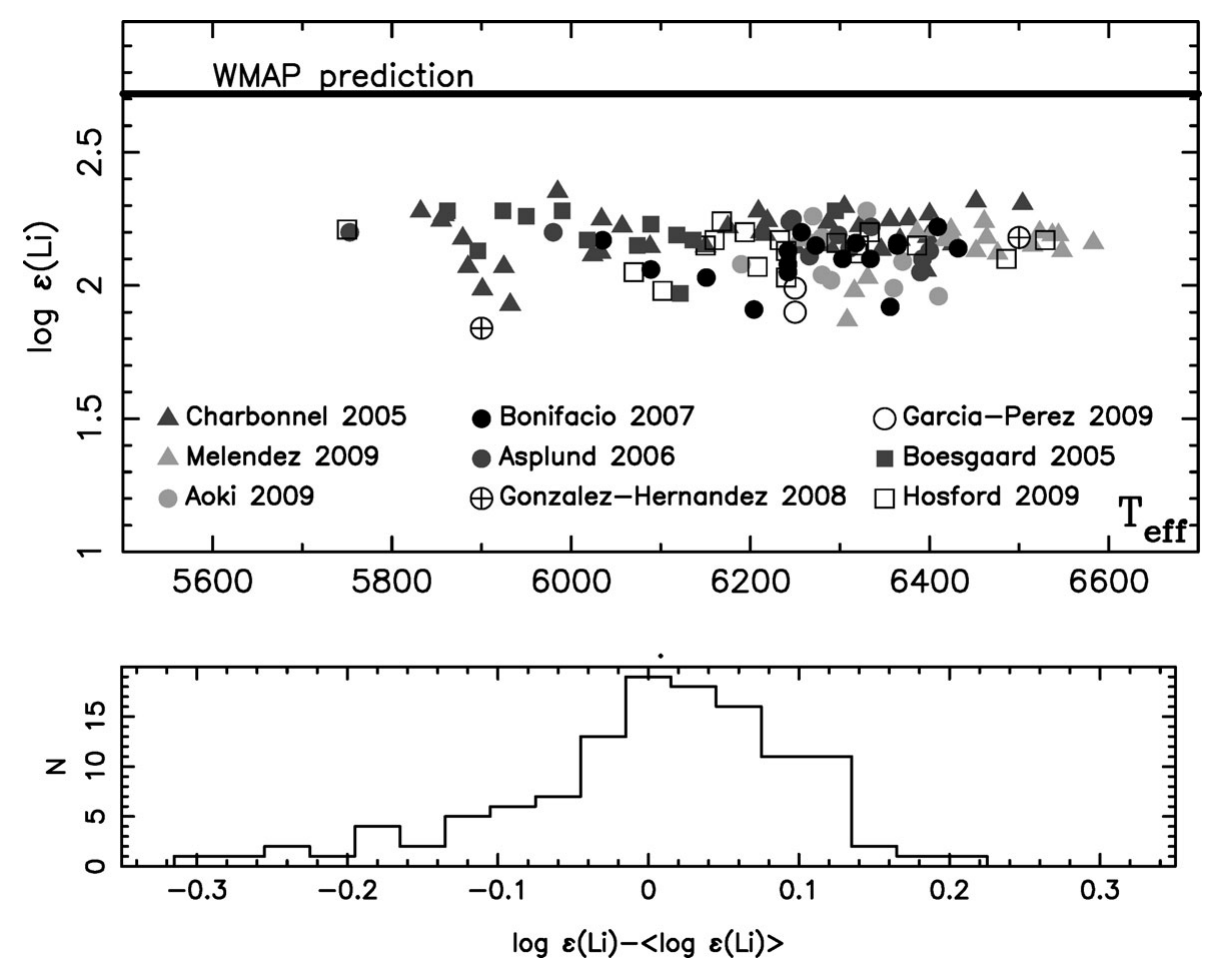

Figure 1. Lithium abundance versus Temperature for metal-poor stars $([\mathrm{Fe} / \mathrm{H}]<-2)$ and (below) histogram. Triangles indicate that the temperature has been determined from photometry, circles from the profile of the hydrogen lines and squares from the excitation temperature. In this interval of temperature and metallicity, the lithium abundance is independent of the temperature but the scatter is rather large. The mean value of the lithium abundance is $\log \epsilon(\mathrm{Li}) \approx 2.15$, more than 0.55 dex below the prediction of standard BB + WMAP (full black line). On the histogram showing the distribution of the distances of each point to the mean value of the lithium abundance it can be seen that the distribution is not gaussian.

In Fig. 1 we present $\log \epsilon(\mathrm{Li})$ versus $\mathrm{T}_{\text {eff }}$, for the metal-poor dwarfs and turnoff stars with $[\mathrm{Fe} / \mathrm{H}]<-2.0$, measured in these different works. There is a rather good agreement between the different determinations, (even if some slight systematic differences appear between different authors). No significant trend of $\log \epsilon(\mathrm{Li})$ vs. $\mathrm{T}_{\text {eff }}$ is apparent. The mean value of the lithium abundance is $\log \epsilon(\mathrm{Li}) \approx 2.15$ : about four times less than the abundance formed by the standard Big Bang nucleosynthesis.

This mean value of the lithium abundance measured in the old galactic stars is also very close to the value measured in $\omega$ Cen (see Bonifacio et al., this symposium): $\log \epsilon(\mathrm{Li}) \approx 2.19 \pm 0.14$. The cluster $\omega$ Cen is now considered as the remnant of a captured dwarf galaxy. As a consequence $\log \epsilon(\mathrm{Li})=2.15$ could be a "universal" value of stellar $\mathrm{Li}$ abundance at low metallicity.

In Fig. 1 the scatter around the mean value of the lithium abundance is 0.10 dex. This scatter is not symmetric (see the histogram in Fig. 1) and is larger than the measurement errors. It is NOT mainly due to systematic differences between different authors, it exists even for the data of a given author.

What is the cause of this scatter of the lithium abundance when $[\mathrm{Fe} / \mathrm{H}]<-2$ ?

If we plot $\log \epsilon(\mathrm{Li})$ versus $[\mathrm{Fe} / \mathrm{H}]$ (Fig. 2) a slight decrease of $\epsilon(\mathrm{Li})$ with the metallicity appears. As a consequence the scatter observed in Fig 1 should be partly due to a 


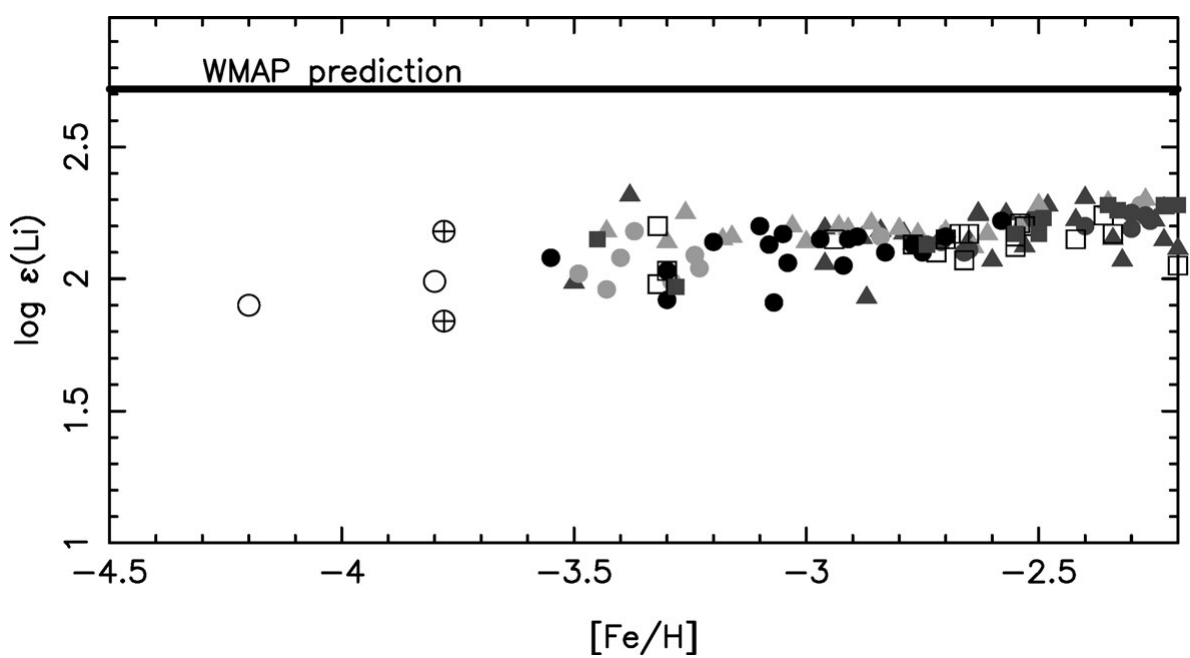

Figure 2. $\log \epsilon(\mathrm{Li})$ vs. $[\mathrm{Fe} / \mathrm{H}]$, for turnoff stars $\left(\mathrm{T}_{\mathrm{eff}}>5800 \mathrm{~K}\right)$. The symbols are the same as in Fig. 1. The slight decline of $\log \epsilon(\mathrm{Li})$ when the metallicity decreases appears for $[\mathrm{Fe} / \mathrm{H}]<-3$.

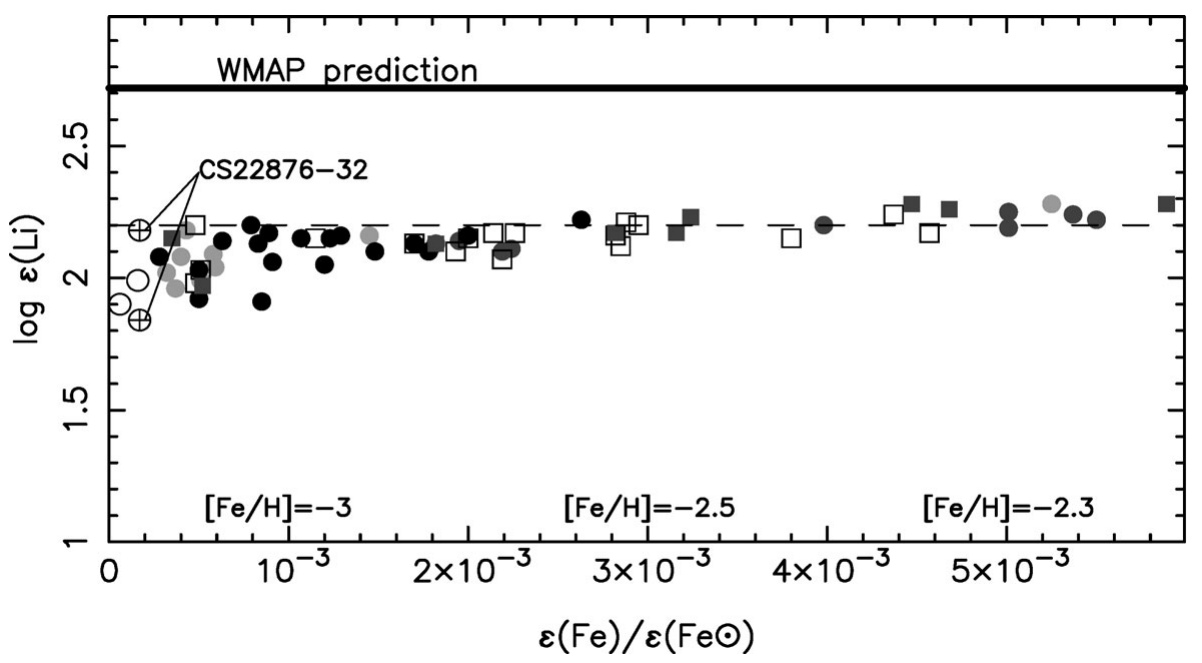

Figure 3. $\log \epsilon(\mathrm{Li})$ vs. the iron abundance $\epsilon\left(\mathrm{Fe}_{*}\right) / \epsilon\left(\mathrm{Fe}_{\odot}\right)$ (linear scale) for turnoff stars $\left(\mathrm{T}_{\mathrm{eff}}>5800 \mathrm{~K}\right)$. The symbols are the same as in Fig. 1. To improve the homogeneity, we have kept only the measurements of the lithium abundance based on a temperature determination independent of the reddening ( $\mathrm{T}_{\mathrm{Hydrogen}}$ or $\left.\mathrm{T}_{\text {exc }}\right)$. When $\epsilon\left(\mathrm{Fe}_{*}\right) / \epsilon\left(\mathrm{Fe}_{\odot}>0.001([\mathrm{Fe} / \mathrm{H}]>-3)\right.$, the scatter around the mean curve is very small $(0.05 \mathrm{dex})$ but at a lower metallicity the scatter suddenly increases. The lithium abundances in the two components of the extremely metal-poor binary CS 22876-32 are different by a factor of two. Lithium would sometimes suffers an extra depletion in the atmospheres of the most metal-poor stars. Then, the pristine value of the lithium abundance would correspond to the higher envelope, $\log \epsilon(\operatorname{Li}) \approx 2.2$.

dependence of lithium abundance on metallicity. This decrease suggests that the extrapolated abundance of lithium could be even lower than 2.15 when extrapolating toward zero-metal stars (just after the Big Bang).

In Fig. 3 we have plotted $\log \epsilon(\mathrm{Li})$ versus $\epsilon(\mathrm{Fe})_{*} / \epsilon(\mathrm{Fe})_{\odot}$ (linear scale), and for a better homogeneity, we have kept only stars with a temperature determined spectroscopically ( $\mathrm{H} \alpha$ profiles or excitation temperature) and thus independent of the interstellar 
reddening. In the interval $2 \times 10^{-3}<\epsilon(\mathrm{Fe})_{*} / \epsilon(\mathrm{Fe})_{\odot}<6 \times 10^{-3}$ the lithium abundance increases steadily and the scatter around the mean curve is extremely small: 0.05 dex.

In the most metal-poor stars $\left(\epsilon(\mathrm{Fe})_{*} / \epsilon(\mathrm{Fe})_{\odot}<2 \times 10^{-3}\right.$, i.e. $[\mathrm{Fe} / \mathrm{H}]<-2.7$, the mean value of the lithium abundance decreases suddenly to reach $\log \epsilon(\mathrm{Li}) \approx 2.0$ dex at $\epsilon(\mathrm{Fe})=0$. (zero-metal stars). Therefore $\log \epsilon(\mathrm{Li}) \approx 2.0$ could be the mean value of the lithium abundance in the early Galaxy. But in this region the scatter at a given metallicity increases strongly (see Sbordone et al. 2010 and this symposium, for confirmation by additional data). This scatter could be interpreted as the result of a variable depletion, decreasing the lithium abundance from a natal value of about $\log \epsilon(\mathrm{Li})=2.2$ dex, this value would represent the pristine value of the lithium abundance in the early Galaxy.

In both cases the pristine value of the lithium abundance is well below the cosmological value derived from the standard Big Bang (with the WMAP value of the $\eta$ parameter).

In Fig 3, no stars are found above $\log \epsilon(\mathrm{Li})=2.2$ dex for $[\mathrm{Fe} / \mathrm{H}]<-2.7$ and above $\log \epsilon(\mathrm{Li})=2.3$ dex for $-2.7<[\mathrm{Fe} / \mathrm{H}]<-2.2$ defining an upper envelope of the lithium abundance. (A handful of Li-rich halo stars have been found in the literature, but all are several times more metal-rich than the stars considered here.)

Several interpretations can be put forward for explaining the gap between the cosmological and the observed lithium and also the sudden scatter appearing at low metallicity.

\subsection{Astration}

It has been proposed that the "uniform" lithium abundance in halo dwarfs could be due to a general process affecting the whole Milky Way lowering the cosmological Li abundance down to the pristine abundance. Piau et al. (2006) have proposed an early astration in very masssive stars Pop III stars (destroying Li). They could have destroyed $3 / 4$ of the primordial lithium, $50 \%$ of the mass of the Galactic halo having been processed in these massive stars.

The scatter of the lithium abundance appearing in the most metal-poor stars could then be explained by a still incomplete mixing of the matter in the Galaxy, or by the fact that these most metal-poor stars would have been accreted from different faint dwarf galaxies or satellites (Frebel et al. 2010) with different astration histories. There are objections to a strong initial astration in our Galaxy: the ejecta of the stars destroying Li would have induced (Prantzos 2007) a large early abundance of elements (e.g. C and O) that is not observed. But, in $\omega$ Cen, remnant of an accreted dwarf galaxy, the lithium abundance $(\log \epsilon(\mathrm{Li}) \approx 2.19$, Bonifacio et al., this symposium), is very similar to the Galactic value for stars of the same metallicity, although they certainly had different astration histories. Probably, a very early global astration of the matter of the universe, by massive first stars, before the formation of the galaxies, would explain the plateau, but would encounter a similar objection about the early abundances of $\mathrm{C}$ and $\mathrm{O}$ in the Galaxy. However it could be possible that these massive Pop III stars rotate and thus eject only external layers with hydrogen and helium (Meynet, this symposium).

\subsection{Depletion in the stellar atmospheres}

The gap between the theoretical value of the lithium abundance predicted by the standard Big Bang and the observed value in the atmosphere of the stars, can be also the result of a depletion of lithium, even in the warm metal-poor dwarfs.

The stellar abundance now observed could be due to diffusion (Michaud et al., 1984). Some calculations have been made (e.g. Richard et al., 2005): a depletion such as figured in Fig, 3, could be matched by a diffusion carefully moderated by some turbulence. The very low scatter of the data around the mean $(0.05 \mathrm{dex})$ in the interval $2 \times 10^{-3}<$ 
$\epsilon(\mathrm{Fe})_{*} / \epsilon(\mathrm{Fe})_{\odot}<6 \times 10^{-3}$ brings severe constaints to the theoretical computations. Also the sudden downward scatter, at the left part of the Figure 3 needs to be explained.

The computation of lithium depletion by diffusion in a globular cluster (Korn et al., 2007) carefully combined with turbulence, shows that the lithium depletion amounts to about $0.26 \mathrm{dex}$. If this correction is applied to the most metal-poor field stars the pristine (or natal) $\mathrm{Li}$ abundance of these stars should be (in the best case) about 2.46 dex, a value still significantly smaller than the cosmological value. As a consequence the depletion by diffusion moderated by turbulence cannot explain completely the gap. Moreover this depletion by diffusion/turbulence in globular clusters is questionned by González Hernández et al. (this symposium).

We have to remark (Fig. 2 and 3 ) that the two components of the extremely metalpoor $([\mathrm{Fe} / \mathrm{H}] \approx-3.8)$ binary star CS 22176-32, analyzed by González Hernández et al. (2008) have a lithium abundance different by a factor of two, although their temperature is higher than $5800 \mathrm{~K}$ and that, as a consequence, no depletion of lithium is expected in these stars. This difference is above the measurement errors. The two stars were presumably born with the same lithium abundance and thus the only explanation of this large difference is that lithium has been depleted (at least) in CS 22176-32B.

"Extra-scatter" by "extra depletion" in the extremely metal-poor stars? This "extradepletion" would appear only, and sometimes, at very low metallicity $([\mathrm{Fe} / \mathrm{H}]<-3)$.

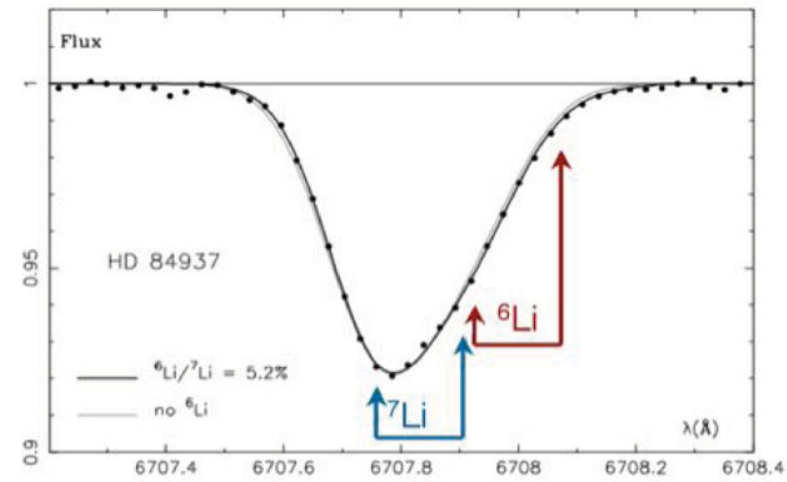

Figure 4. Profile of the doublets of ${ }^{7} \mathrm{Li}$ and ${ }^{6} \mathrm{Li}$ in a very metal poor turnoff star HD 84937, compared to a synthetic spectrum computed with no ${ }^{6} \mathrm{Li}$ and ${ }^{6} \mathrm{Li} /{ }^{7} \mathrm{Li}=5 \%$

\section{Observation of ${ }^{6} \mathrm{Li}$ in the most metal poor stars in the Milky Way}

Since ${ }^{6} \mathrm{Li}$ is not significantly formed by the standard Big Bang, if ${ }^{6} \mathrm{Li}$ is observed in very metal-poor stars it is supposed to be formed mainly by cosmic rays.

If ${ }^{6} \mathrm{Li}$ exists in these stars, its abundance is very small and it is thus very difficult to detect, in particular because the lines (doublets) of ${ }^{6} \mathrm{Li}$ and ${ }^{7} \mathrm{Li}$ are overlapping (see Fig. 4). Very high resolution and high $\mathrm{S} / \mathrm{N}$ spectra are required as also very precise computations of the profiles.

Several groups have recently tried to measure ${ }^{6} \mathrm{Li}$ in metal-poor halo stars (see in particular Asplund et al. 2006, Asplund \& Meléndez 2008, Cayrel et al. 2007, García Pérez et al. 2009).

Asplund et al. $(2006,2008)$ observed a sample of 27 turnoff stars with $-3.3<[\mathrm{Fe} / \mathrm{H}]<$ -1.2. In twelve of them, they detected ${ }^{6} \mathrm{Li}$. The abundance of ${ }^{6} \mathrm{Li}$ was constant $\log \epsilon\left({ }^{6} \mathrm{Li}\right) \approx$ 
0.8 dex and the ratio ${ }^{6} \mathrm{Li} /{ }^{7} \mathrm{Li}$ was between 4 and $10 \%$. In all the 27 stars the abundance of lithium or its upper limit is compatible with a plateau with ${ }^{6} \mathrm{Li} /{ }^{7} \mathrm{Li} \approx 5 \%$.

This result remains fragile: Cayrel et al. (2007) have shown that, if a classical analysis of a given star would provide a ratio ${ }^{6} \mathrm{Li} /{ }^{7} \mathrm{Li}=4 \%$ the result would be ${ }^{6} \mathrm{Li} /{ }^{7} \mathrm{Li}=0 \%$ if the asymetry of the lines, due to convection, is taken into account (3D computations).

Recently, García Pérez et al. (2009) have insisted on the fact that the error of the ${ }^{6} \mathrm{Li}$ measurement is generally underestimated due to the uncertainty of the position of the continuum, the residual fringes etc. It is interesting to remark that Asplund \& Meléndez (2008) found that the ${ }^{6} \mathrm{Li} /{ }^{7} \mathrm{Li}$ ratio in G64-37 is $11 \%$ while García Pérez et al. (2009) found less than $1 \%$.

\subsection{Have we really observed ${ }^{6}$ Li in EMP stars?}

In none of the stars, the ${ }^{6} \mathrm{Li}$ isotope is detected with a precision higher or equal to $3 \sigma$ (Steffen et al., this symposium). If the error is a little underestimated, and if there is in fact, no ${ }^{6} \mathrm{Li}$ in the old metal-poor stars, the noise would mimic an absorption of ${ }^{6} \mathrm{Li}$ in about half of the stars (detection) and an emission of ${ }^{6} \mathrm{Li}$ in the other half of the stars (no detection). This is not far from what is observed...

More precise computations (NLTE, 3D) about spectra with higher $\mathrm{S} / \mathrm{N}$ ratios and a better defined continuum are necessary to firmly conclude about the detection and the abundance of ${ }^{6} \mathrm{Li}$ in the most metal-poor stars.

\section{Conclusion : Is it possible to explain the behaviour of ${ }^{6} \mathrm{Li}$ and ${ }^{7} \mathrm{Li}$ in the early Galaxy?}

$$
\text { 5.1. }{ }^{6} \mathrm{Li}
$$

The absence of ${ }^{6} \mathrm{Li}$, or at least an abundance below the limit of detection, would be easy to explain since the quantity of ${ }^{6} \mathrm{Li}$ formed by the standard Big Bang and by the cosmic rays is supposed to be very low.

The measurement of the ${ }^{6} \mathrm{Li}$ abundance is difficult. If some ${ }^{6} \mathrm{Li}$ is observed, it should have been formed (before the birth of our old turnoff stars) by Galactic Cosmic Rays or during the explosion of massive supernovae in superbubbles: we should then observe a clear increase of ${ }^{6} \mathrm{Li}$ with $[\mathrm{Fe} / \mathrm{H}]$ like the increase of boron and beryllium (see Boesgaard 2004, Prantzos 2007). At the present time, a large abundance of ${ }^{6} \mathrm{Li}$, more or less constant with $[\mathrm{Fe} / \mathrm{H}]$, such as observed by Asplund et al. $(2006,2008)$ : $\log \epsilon(\mathrm{Li})=0.8 \mathrm{dex}$, cannot be understood in the frame of the standard theories.

\section{2. ${ }^{7} \mathrm{Li}$}

Depending on the interpretation of Fig. 3, the pristine stellar abundance of ${ }^{7} \mathrm{Li}$ in our Galaxy is $\log \epsilon(\mathrm{Li})=2.0$ or 2.2 dex: in both cases, it is far from the value $(2.72 \mathrm{dex})$ of the ${ }^{7} \mathrm{Li}$ abundance produced by the standard Big Bang nucleosynthesis, according to the WMAP specifications. Some explanations can be proposed none of them completely satisfactory.

- Early astration - According to Piau et al. (2006), the primordial ${ }^{7} \mathrm{Li}$, (such as computed by the Big Bang) would have been severely destroyed in the Galaxy before the formation of the old metal-poor stars by astration of the matter in massive Pop III stars, lowering the lithium abundance to the observed pristine abundance. But this theory cannot explain that the same pristine abundance of lithium is observed in the Milky Way and in $\omega$ Cen (the remnant of an accreted dwarf galaxy) which certainly had different astration histories (see section 3.1). Moreover in this case a strong excess (not observed) 
of $\mathrm{C}$ and $\mathrm{O}$ in the matter of the early Galaxy would be, a priori, expected, However it has been remarked (Meynet, this symposium) that massive rotating Pop III stars would eject only external layers with hydrogen and helium (without excess of $\mathrm{C}$ and $\mathrm{O}$ ).

- Depletion / Diffusion - A second hypothesis is that lithium has been depleted in the atmosphere of the observed turnoff stars during their long evolution, by atomic diffusion carefully partially compensated by turbulence (see section 3.2). The data gathered in Fig. 3, which show for $[\mathrm{Fe} / \mathrm{H}]>-3$ a very small scatter (entirely explained by the determination errors) are a challenge to the computations of such an uniform depletion. The downward scatter of the lithium abundance in the extremely metal-poor stars remains to be explained (variable extra-depletion ?), as well as the absence (within our limits of $\mathrm{T}_{\text {eff }}$ and metallicity) of any (Li-rich) star in the "desert" between the high Big Bang lithium abundance and the plateau. Anyway, if the "depletion correction" computed by Korn et al. (2007) for the globular cluster NGC 6397 is applied to the field metal-poor dwarfs, the resulting value of the pristine lithium abundance (in the best case, about 2.46 dex) is again far from the cosmological predictions.

- Gravity waves - The behaviour of lithium should be evaluated taking into account the gravity waves (e.g. Talon \& Charbonnel 2004, see also Talon et al., this symposium): the results of this promising theory are eagerly awaited.

- Uncertainties in the Big Bang theories - The production of ${ }^{7} \mathrm{Li}$ in the Big Bang could be lower in the standard nucleosynthesis if a resonance level (Cyburt \& Pospelov, 2009 ) is taken ito account in the transformation of ${ }^{7} \mathrm{Be}$ into ${ }^{9} \mathrm{~B}$.

Moreover, a number of theories have been proposed for various more or less ad hoc non-standard Big Bang nucleosyntheses (see e.g. Iocco et al. 2009).

As a conclusion, in addition to progresses in fundamental physics and nucleosynthesis, the use of NLTE, 3D model atmospheres for interpreting high quality spectra, analysing differential observations (comparison dwarfs-subgiants, binaries, delicate trends, small scatters and other such details) should shed some light on the always complex problem of the lithium abundance in the early Galaxy.

\section{Acknowledgments}

This text benefitted from conversations with P. Bonifacio, L. Sbordone and E. Caffau.

\section{References}

Aoki, W., Barklem, P. S., Beers, T. C., Christlieb, N., Inoue, S. et al. 2009, ApJ, 698, 1803 Alonso, A., Arribas, S., \& Martinez-Roger, C. 2009, A\&A, 313, 873

Asplund, M., Lambert, D. L., Nissen, P. E., Primas, F., \& Smith, V. V. 2006, ApJ, 644, 229

Asplund, M. \& Meléndez, J. 2008, AIP Conf. Proc. FIRST STARS III: First Stars II Conference, Vol. 990, p. 342

Bennett, C. L. et al. 2003, ApJS, 148, 1

Boesgaard, A. M. 2004, Carnegie Obs. Astrophys. Ser., Vol. 4: Origin and Evolution of the Elements, eds. A. McWilliam and M. Rauch (Cambridge: Cambridge Univ. Press)

Boesgaard, A. M., Stephens, A., \& Deliyannis, C. P. 2005, ApJ, 63, 398

Bonifacio, P., Molaro, P., Sivarani, T., Spite, M., Spite, F. et al. 2007, A\&\&A, 462, 851

Bonifacio, P. \& Molaro, P. 1997, MNRAS, 285, 847

Cayrel, R., Spite, M., Spite, F., Vangioni-Flam, E., Cassé, M., \& Audouze, J. 1999, A $\& A, 343$, 923

Cayrel, R., Steffen, M., Chand, H., Bonifacio, P., Spite, M., Spite, F. et al. 2007, A\&A, 473, L37

Charbonnel, C. \& Primas, F. 2005, A\&AA, 442, 961 
Cyburt, R. H., Fields, B. D., \& Olive, K. A. 2008, JCAP, 11, 12

Cyburt, R. H. \& Pospelov, M. 2009, arXiv(0906.4373)

Deliyannis, C. P., Demarque, P., \& Kawaler, S. D. 1990 ApJS, 73, 21

Frebel, A., Simon, J. D., Geha, M., \& Willman, B. 2010 ApJ, 708, 560

García Pérez, A. E., Aoki, W., Inoue, S., Ryan, S. G., Suzuki, T. K. et al. 2009, A\&A, 504, 213

García Pérez, A. E., Christlieb, N., Ryan, S. G., Beers, T. C. et al. 2008, Physica Scripta, 133, 4036

González Hernández, J., Bonifacio, P., Ludwig, H.-G., Caffau, E., Spite, M., Spite, F. et al. 2008, $A \mathscr{E} A, 480,233$

Hobbs, L. M. \& Thorburn, J. A. 1991, ApJ, 375, 116

Hosford, A., Ryan, S. G., García Pérez, A. E., Norris, J. E., \& Olive, K. A. 2009, A $\& A$, 493, 601

Iocco, F., Mangano, G., Miele, G., Pisanti, O., \& Serpico, D. 2009, Phys. Rep., 472, 1

Komatsu, E., Dunkley, J., Nolta, M. R. et al. 2009, ApJS, 180, 330

Korn, A. J., Grundahl, F., \& Richard, O. 2007, ApJ, 671, 402 (Paper I)

Meléndez, J., Casagrande, L., Ramírez, I., \& Asplund, M. 2010, Proceedings of the IAU symp. 265 "Chemical Abundances in the Universe: Connecting First Stars to Planets", K. Cunha, M. Spite \& B. Barbuy, eds., Cambridge University Press, p. 71

Michaud, G., Fontaine, G., \& Beaudet, G. 1984, ApJ, 282, 206

Molaro, P., Primas, F., \& Bonifacio, P. 1995, A\&A, 348, 211

Nissen Poul, E., Lambert, D. L., Primas, F., \& Smith, V. V. 1999, A\& A, 295, 47

Piau, L., Beers, T. C., Balsara, D. S., Sivarani, T., Truran, J. W., \& Ferguson, J. W. 2006, ApJ, 653,300

Pinsonneault, M. H., Deliyannis, C. P., \& Demarque, P. 1992, ApJS, 78, 179

Prantzos, N. 2007, Space Sci. Rev., 130, 27

Richard, O., Michaud, G., \& Richer, J. 2005, ApJ, 619, 538

Sbordone, L., Bonifacio, P., Caffau, E., Ludwig, H.-G., Behara, N. et al. 2010, Proceedings IAU Symposium No. 265, "Chemical Abundances in the Universe: Connecting First Stars to Planets", K. Cunha, M. Spite \& B. Barbuy, eds., Cambridge University Press, p. 75

Smith, V. V., Lambert, D. L., \& Nissen Poul, E. 1998, ApJ, 408, 262

Smith, V. V., Lambert, D. L., \& Nissen Poul, E. 1998, ApJ, 506, 405

Spergel, D. N., Bean, R., Doré, O. et al. 2007, ApJS, 170, 377

Spite, M. \& Spite, F. 1982a, Nature, 297, 483

Spite, F. \& Spite, M. 1982b, A\& A, 115, 357

Spite, F., Spite, M. \& Maillard, J. P. 1984, A\&A, 141, 56

Spite, M., Francois, P., Nissen, P. E., \& Spite, F. 1996, A\&\&A, 307, 172

Steffen, M., Cayrel, R., Bonifacio, P., Ludwig, H.-G., \& Caffau, E. 2010, Proceedings of the IAU symp. 265 "Chemical Abundances in the Universe: Connecting First Stars to Planets", K. Cunha, M. Spite \& B. Barbuy, eds., Cambridge University Press, p. 23

Talon, S. \& Charbonnel, C. 2004, A\&A, 418, 1051 